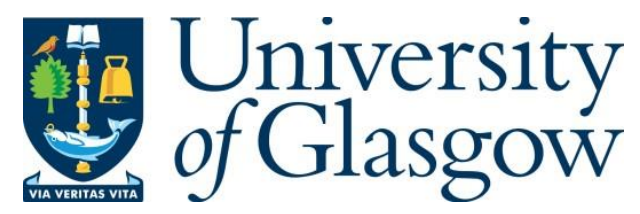

Zhang, L., Ijaz, A., Xiao, P., Quddus, A. and Tafazolli, R. (2016) Single-Rate and MultiRate Multi-Service Systems for Next Generation and Beyond Communications. In: 2016 IEEE 27th Annual International Symposium on Personal, Indoor, and Mobile Radio Communications (PIMRC), Valencia, Spain, 04-08 Sep 2016, ISBN 9781509032549.

There may be differences between this version and the published version. You are advised to consult the publisher's version if you wish to cite from it.

http://eprints.gla.ac.uk/143642/

Deposited on: 6 July 2017

Enlighten - Research publications by members of the University of Glasgow http://eprints.gla.ac.uk 


\title{
Single-rate and Multi-rate Multi-service Systems for Next Generation and Beyond Communications
}

\author{
Lei Zhang, Ayesha Ijaz, Pei Xiao, Atta Quddus and Rahim Tafazolli \\ Institute for Communication Systems (ICS) \\ University of Surrey \\ Guildford, GU2 7XH, UK \\ Email: \{lei.zhang, a.ijaz, p.xiao, a.quddus, r.tafazolli\}@ surrey.ac.uk
}

\begin{abstract}
To flexibly support diverse communication requirements (e.g., throughput, latency, massive connection, etc.) for the next generation wireless communications, one viable solution is to divide the system bandwidth into several service subbands, each for a different type of service. In such a multi-service (MS) system, each service has its optimal frame structure while the services are isolated by subband filtering. In this paper, a framework for multi-service (MS) system is established based on subband filtered multi-carrier (SFMC) modulation. We consider both single-rate (SR) and multi-rate (MR) signal processing as two different MS-SFMC implementations, each having different performance and computational complexity. By comparison, the SR system outperforms the MR system in terms of performance while the MR system has a significantly reduced computational complexity than the SR system. Numerical results show the effectiveness of our analysis and the proposed systems. These proposed SR and MR MS-SFMC systems provide guidelines for next generation wireless system frame structure optimization and algorithm design.
\end{abstract}

\section{INTRODUCTION}

The aims of wireless communications, from the 1st generation to the 5 th generation $(5 \mathrm{G})$ and beyond, have consistently shifted from mere provision of voice telephony to data connectivity to the provision of multiple services (MS) from connected devices as well as connected people. The technical challenges are several-fold: ultra-high user/device density (up to $1 \mathrm{million} / \mathrm{km}^{2}$ ), very high data rates (gigabits/second), extreme reliability $(>99.999 \%)$ and low end-to-end latency $(<1$ millisecond) [1], [2]. These demanding requirements make the all-in-one air-interface and frame structure design for future networks extremely challenging. For example, the MachineType-Communications (e.g., smart meters, eHealth devices, etc.) require smaller subcarrier spacing (i.e., larger symbol duration) to support massive delay tolerant devices. On the other hand, tactile and autonomous vehicles communications have very stringent latency requirements, which need significantly reduced symbol duration (i.e., increased subcarrier spacing). However, separate radio designs for separate services make the operation and management of the system highly complex, expensive and inefficient. One viable solution is to divide the system bandwidth into several service bands, each for a different type of service [3], [4], [5]. Such an MS system is shown in Fig. 1, where a specifically optimized frame structure is designed for different types of services, with (or without) a certain guard band (GB) between them to mitigate the interference.

Mathematically, combining multiple frame structures with different subcarrier spacings in one frequency band would destroy the orthogonality of an multi-carrier system, resulting in inter-service-band-interference (ISBI). Moreover, waveform is also a key factor to determine the interference level. Orthogonal frequency division multiplexing (OFDM) [6] is NOT suitable for this MS system due to the high ISBI caused by its high out of band (OoB) emission level. Several new waveforms are proposed to address the problem, such as universal filtered multi-carrier (UFMC) [3], [7], [8], [9], [10], filtered OFDM (fOFDM) [4], [11], filter bank multi-carrier (FBMC) [12], [13], [14] and generalized frequency division multiplexing (GFDM) [15], etc.

Among these, UFMC and f-OFDM are symbol-based subband filtered multi-carrier (SFMC) system. They have drawn significant attentions from academia and industry recently. It inherits the advantages of OFDM system such as low complexity and effective one-tap channel equalization. In addition, it also achieves comparable OoB emission as FBMC systems [3], [7], [8]. As one implementation of SFMC, f-OFDM uses long subband filter to relax synchronization requirements [11], [4]. The adjacent symbols overlap to each other in order to reduce the filter tails induced overhead. On the other hand, UFMC system uses short subband filter without symbol overlapping. In this paper, we specify MS-SFMC as f-OFDM based MS system. However, the results can be extended into UFMC based implementation straightforwardly.

The system and algorithm design for SFMC system was focused on the single service case in the literature [3], [7], [8]. In [4], the authors considered single-rate (SR) multi-service system for performance comparisons with UFMC based waveform on simulations. In this paper, we will systematically model two implementations of the MS-SFMC system, i.e., SR MS-SFMC and MR (multi-rate) MS-SFMC. In the SR MSSFMC system, the baseband sampling rate is fixed and keeps the same during the processing for all service bands. On the other hand, MR MS-SFMC has variable sampling rate in the baseband signal processing to take the advantage of using lowcomplexity low-dimension Discrete Fourier Transform (DFT). The system models are derived for both SR and MR MSSFMC systems with discussions on the pros and cons of the 


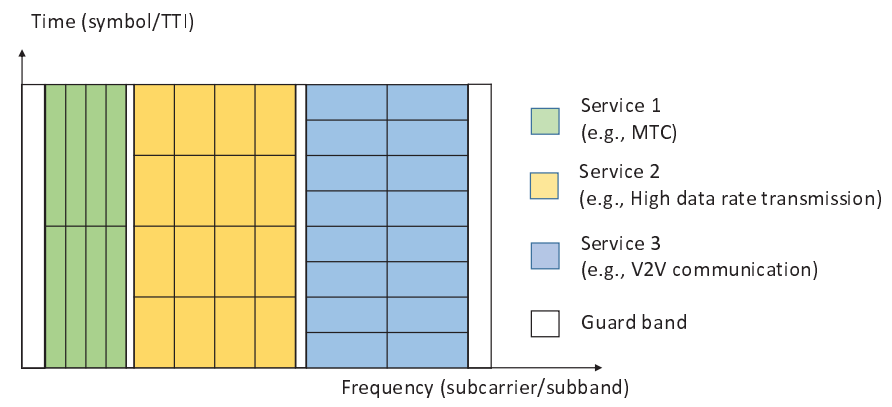

Fig. 1. An example of multi-service multi-carrier system.
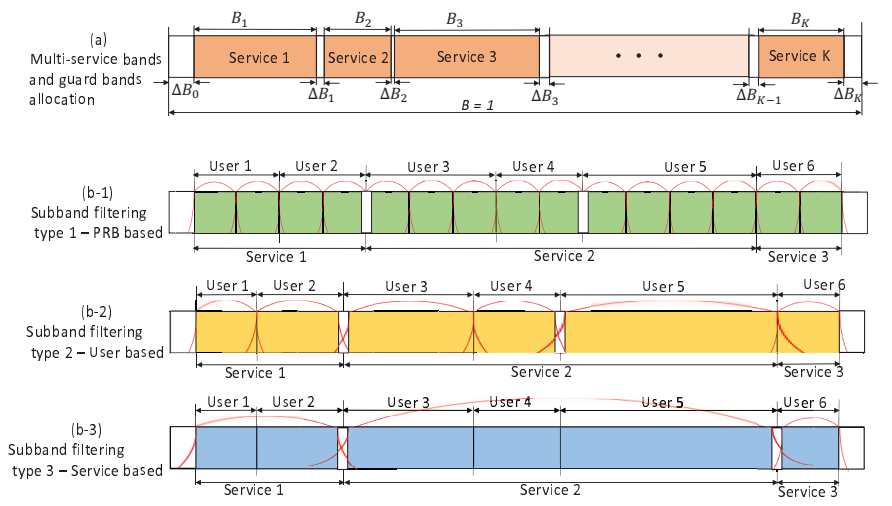

Fig. 2. (a): Service bands and guard bands allocation; (b): Three types of subband filtering methods. (note that the number of user, subband and service are for demonstration purpose only in this figure).

two implementations to provide a practical selection guidance for MS system. In addition, these implementations provide a basic framework for next generation wireless system frame structure optimization and algorithm design.

The rest of the paper is organized as follows. In Section II, the system models for SR and MR MS-SFMC system are derived separately. The comparisons of SR and MR based implementations in terms of performance and computational complextiy are discussed in Section III. Simulation results are provided in Section IV and conclusions and future works are given in Section V.

\section{MS-SFMC SYSTEMS}

\section{A. Frequency and time domain implementations}

For a system containing multiple types of services, it is natural to assume that each service supports one or more users, while each user can be granted arbitrary number of consecutive or non-consecutive physical resource blocks (PRBs). The MSSFMC system has a 4-tier frequency resource structure: system bandwidth, service bandwidth, PRB, and subcarrier. Therefore, subbands can be based on either PRBs, user, service, as shown in Fig. 2 (b-1), (b-2) and (b-3), respectively. PRB is the minimum scheduling granularity and the subband filtering based on one PRB can have the largest design flexibility (Fig. 2 (b-1)). On the other hand, this implementation also incurs the highest computational complexity among all due to dense subband filtering operation. On the contrary, service based subband filtering method (Fig. 2 (b-3)) has the lowest computational complexity and the users (and PRBs) in one service share the same filter design parameters. However, different users in one service may suffer from different channel qualities, receiver sensitivities, etc. which can not be optimized by filter design independently. In the middle, user-based subband filtering as shown in Fig. 2 (b-2) is a compromised solution between PRB-based and service-based methods. Note that PRB-based implementation is the most general case, and is thus the case considered in this paper.

In the time domain, the symbol durations among services are different due to the different subcarrier spacings. For example, without consider the guard interval between symbols, two services with subcarrier spacing $\Delta f_{1}=2 \Delta f_{2}$ implies that the symbol duration has the relationship of $\Delta T_{2}=2 \Delta T_{1}$. In principle, different services can have arbitrary symbol duration, resulting in totally non-synchronized MS system. However, in practice, it is beneficial to design an MS system with an integral least common multiplier (LCM) symbol duration $T_{L C M}$ for all services, i.e.,

$$
L_{L C M}=N_{\text {sym }, 1} L_{1}=\cdots=N_{\text {sym }, K} L_{K} .
$$

where $L_{k}$ is the $k$-th service symbol duration in samples and $L_{L C M}$ refers to the total number of samples in one LCM symbol. $N_{\text {sym }, 1}, N_{\text {sym }, 2} \cdots, N_{\text {sym }, K}$ are non-zero integers. For the MS system satisfy equation (1), we call it is generally synchronized (GS). One LCM symbol for a GS system is a closed space that does not affect and also is not affected by other symbols out of the considered LCM symbol. A GS system simplifies system and algorithms design and performance analysis since only limited symbols need to be considered in a processing window.

\section{B. MS system and signal parameters}

Let us consider an MS communication system with system bandwidth $B$ offering total $K$ different services. The $k$-th service bandwidth is $B_{k}$ and the GB between the $k$-th and the $(k+1)$-th service is $\Delta B_{k}$. In addition, we assume $\Delta B_{0}$ and $\Delta B_{K}$ at edges of the frequency band are GBs (or unoccupied bands) between the considered MS-SFMC system and other systems. The bandwidth allocation is shown in Fig. 2 (a). Without loss of generality, we assume the system bandwidth $B$ is normalized to unity, i.e.,

$$
B=\sum_{k=0}^{K} \Delta B_{k}+\sum_{k=1}^{K} B_{k}=1 .
$$

Let us use $M_{k}$ as the number of subcarriers for the $k$-th service in an MS-UFMC system and the $k$-th service contains $V_{k}$ subbands with each subband consisting of equal number of subcarriers $Z_{k}$, i.e., $M_{k}=Z_{k} V_{k}$. In total, the system has $V=\sum_{i=1}^{K} V_{k}$ subbands. 


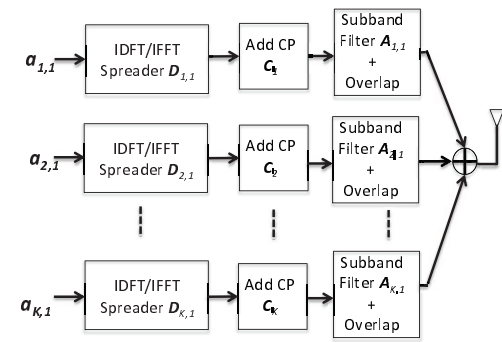

(a): SR MS-SFMC Transmitter

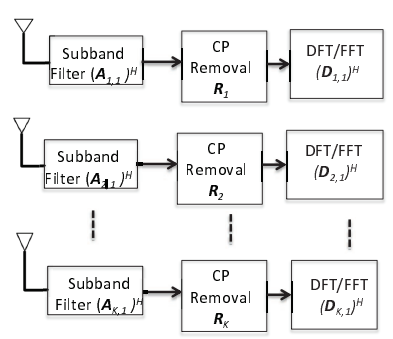

(b): SR MS-SFMC Receivers
Fig. 3. Transmitter and receiver block diagram of SR MS-SFMC systems (each service contains only one subband in this diagram for brevity).

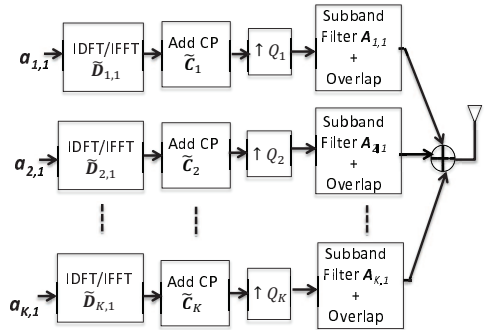

(a): MR MS-SFMC Transmitte

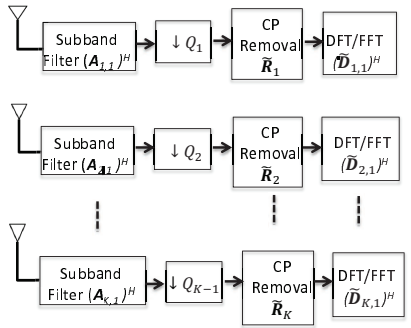

(b): MR MS-SFMC Receivers
Fig. 4. Transmitter and receiver block diagram of MR MS-SFMC systems (each service contains only one subband in this diagram for brevity).

For one LCM symbol duration, it can transmit $N_{\text {info }}=$ $\sum_{i=1}^{K} N_{\text {sym }, k} M_{k}$ modulated information symbols. Writing it in vector form

$$
\mathbf{a}=\left[\mathbf{a}_{1} ; \mathbf{a}_{2} ; \cdots ; \mathbf{a}_{K}\right] \in \mathbb{C}^{N_{i n f o} \times 1}
$$

with

$$
\mathbf{a}_{k}=\left[\mathbf{a}_{k, 1} ; \mathbf{a}_{k, 2} ; \cdots ; \mathbf{a}_{k, V_{k}}\right] \in \mathbb{C}^{N_{s y m, k} M_{k} \times 1}
$$

being the information symbols transmitted in the $k$-th service in one LCM symbol. $\mathbf{a}_{k, i}=\left[\mathbf{a}_{k, v, 1} ; \mathbf{a}_{k, v, 2} ; \cdots ; \mathbf{a}_{k, v, N_{s y m, k}}\right] \in$ $\mathbb{C}^{N_{\text {sym }, k} Z_{k} \times 1}$ is a vector of symbols transmitted on the $v$ th subband in the $k$-th service in one LCM symbol where $\mathbf{a}_{k, v, l}=\left[a_{k, v, l}(0), a_{k, v, l}(1), \cdots, a_{k, v, l}\left(Z_{k}-1\right)\right]^{T} \in \mathbb{C}^{Z_{k} \times 1}$ denotes the information symbols transmitted in the $l$-th symbol of the LCM symbol and at the $v$-th subband of $k$-th service. We assume that information symbols $a_{k, v, l}(i)$ have zero mean and variance $\rho_{\text {sym }}^{2}$.

\section{SR MS-SFMC system model}

In the SR MS-SFMC system, the baseband sampling rate is fixed and remains the same during the processing for all service bands. The SR MS-SFMC system transmitter and receiver block diagrams are shown in Fig. 3, where the IDFT (inverse DFT) modulation is performed as the first step, followed by cyclic prefix (CP) insertion. The IDFT size for the $k$-th service is

$$
N_{k}=M_{k} / B_{k}
$$

The signal in $i$-th symbol and $v$-th subband of the $k$-th service after the IDFT and CP inserting can be written in matrix form as

$$
\mathbf{b}_{k, v, i}=\mathbf{C}_{k} \mathbf{D}_{k, v} \mathbf{a}_{k, v, i},
$$

where $\mathbf{C}_{k}=\left[\mathbf{0}_{L_{C P, k} \times\left(N_{k}-L_{C P, k}\right)}, \mathbf{I}_{L_{C P, k}} ; \mathbf{I}_{N_{k}}\right] \in \mathbb{R}^{L_{k} \times N_{k}}$ is the matrix form of the $\mathrm{CP}$ insertion with $L_{C P, k}$ being the $\mathrm{CP}$ length for the $k$-th service and $L_{k}=N_{k}+L_{C P, k}$ being the symbol duration of the $k$-th service in samples. Note that the sampling rate in the SR system is the same for all services in the whole baseband signal processing. $\mathbf{D}_{k, v} \in \mathbb{C}^{N_{k} \times Z_{k}}$ is a submatrix of the $N_{k}$-point normalized and frequency shifted IDFT matrix. The element in the $l$-th row and $n$-th column of $\mathbf{D}_{k, v}$ is $d_{l, n}=\frac{1}{\sqrt{N}_{k}} e^{j \cdot 2 \pi l\left(n+\eta_{k, v}\right) / N_{k}}$, where $\eta_{k, v}$ is the frequency shift for the $v$-th subband in the $k$-th service and is given as

$$
\eta_{k, v}=N_{k}\left(\sum_{m=1}^{k-1} B_{m}+\sum_{m=0}^{k-1} \Delta B_{m}\right)+\sum_{m=1}^{V_{k}-1} Z_{k} .
$$

Note that $\mathbf{D}_{k, v} \mathbf{a}_{k, v, i}$ can be implemented by the fast IDFT algorithms when $N_{k}$ is a power of 2 .

Let us assume that the filter for the $v$-th subband of the $k$-th service is $\mathbf{f}_{k, v}=\left[f_{k, v}(0), f_{k, v}(1), \cdots, f_{k, v}\left(L_{F, k}-1\right)\right]$ where $L_{F, k}$ is the filter length for the $k$-th service and we assumed one service uses the same filter length. However, the filter lengths for different services (e.g., $L_{F, k 1}$ and $L_{F, k 2}$ ) are not necessarily the same. Filtering the signal $\mathbf{b}_{k, v, i}$ with the subband filter, the output of the subband filter is

$$
\mathbf{c}_{k, v, i}=\mathbf{f}_{k, v} * \mathbf{b}_{k, v, i}=\mathbf{A}_{k, v} \mathbf{b}_{k, v, i},
$$

where $*$ denotes linear convolution of two vectors and $\mathbf{A}_{k, v} \in \mathbb{C}^{\left(L_{k}+L_{F, k}-1\right) \times L_{k}}$ is a Toeplitz matrix with its first column and first row being $\tilde{\mathbf{f}}_{k, v}=\left[\mathbf{f}_{k, v}, \mathbf{0}_{1 \times\left(N_{k}-1\right)}\right]^{T}$ and $\left[f_{k, v}(0), \mathbf{0}_{1 \times\left(L_{k}-1\right)}\right]$, respectively.

The length of $\mathbf{c}_{k, v, i}$ is $L_{k}+L_{F, k}-1$ samples and the extra $L_{F, k, v}-1$ samples more than the symbol duration are extended to adjacent symbols (i.e., overlapping with adjacent symbols) to reduce the system overhead as shown in Fig. 3. After the overlapping and frame structure construction, the signal in $i$-th symbol and $v$-th subband of the $k$-th service can be written as

$\mathbf{q}_{k, v, i}=\frac{1}{\rho_{k, v}}\left(\overline{\mathbf{A}}_{k, v} \mathbf{b}_{k, v, i}+\tilde{\mathbf{A}}_{k, v} \mathbf{b}_{k, v, i-1}+\hat{\mathbf{A}}_{k, v} \mathbf{b}_{k, v, i+1}\right)$,

where $\rho_{k, v}=\sqrt{\mathbf{e}_{k, v, i}^{H} \mathbf{e}_{k, v, i} / Z_{k}}$ is the transmission power normalization factor with $\mathbf{e}_{k, v, i}=\overline{\mathbf{A}}_{k, v} \mathbf{b}_{k, v, i}+$ $\tilde{\mathbf{A}}_{k, v} \mathbf{b}_{k, v, i-1}+\hat{\mathbf{A}}_{k, v} \mathbf{b}_{k, v, i+1} \cdot \mathbf{b}_{k, v, i-1}$ and $\mathbf{b}_{k, v, i+1}$ are the previous and next SFMC symbols due to the symbols overlapping. $\tilde{\mathbf{A}}_{k, v}=\left[\mathbf{0}_{\left(L_{k}-L_{D N, k, v}\right) \times L_{k}} ; \mathbf{A}_{D N, k, v}\right], \quad \hat{\mathbf{A}}_{k, v}=$ $\left[\mathbf{A}_{U P, k, v} ; \mathbf{0}_{\left(L_{k}-L_{U P, k}\right) \times L_{k}}\right]$ with $\mathbf{A}_{U P, k, v}$ and $\mathbf{A}_{D N, k, v}$ being the first $L_{U P, k}$ and the last $L_{D N, k}$ columns of $\mathbf{A}_{k, v}$, respectively. $\overline{\mathbf{A}}_{k, v}$ is a $L_{k} \times L_{k}$ matrix by taking the $\left(L_{U P, k}+1\right)$-th to $L_{k}-L_{D N, k}$ columns of $\mathbf{A}_{k, v}$. Apparently, $L_{U P, k}+L_{D N, k}=$ $L_{F, k}-1$. The values of $L_{U P, k}$ and $L_{D N, k}$ decide how the symbols overlap to the adjacent symbols. In general, we can overlap the equal number of samples to the previous and the 
next symbols, i.e., $L_{U P, k}=L_{D N, k}=\left(L_{F, k}-1\right) / 2$ for odd $L_{F, k}$ and $L_{U P, k}=L_{F, k} / 2$ and $L_{U P, k}=L_{F, k} / 2-1$ for even $L_{F, k}$.

Due to the adjacent symbols overlapping, the second and third terms in equation (9) are inter-symbol interference terms to the $i$-th symbol and $v$-th subband of the $k$-th service.

Considering that one LCM symbol contains $N_{\text {sym }, k}$ SFMC symbols in the $k$-th service as shown in (1), the $v$-th subband of the $k$-th service can be expressed as

$$
\mathbf{q}_{k, v}=\left[\mathbf{q}_{k, v, 1} ; \mathbf{q}_{k, v, 2} ; \cdots ; \mathbf{q}_{k, v, N_{s y m, k}}\right] .
$$

By considering all subbands and all services in the system, the signal in one LCM symbol before transmitting over the channel can be written as

$$
\mathbf{p}=\sum_{k=1}^{K} \sum_{v=1}^{V_{k}} \mathbf{q}_{k, v} \in \mathbb{C}^{L_{L C M} \times 1} .
$$

Let us assume the channel between the transmitter and the $m$-th user is $\mathbf{h}_{m}=\left[h_{m}(0), h_{m}(1), \cdots, h_{m}\left(L_{C H, m}-1\right)\right]$ where $L_{C H, m}$ is the length of the channel in samples. Using equation (11), the received signal at the $m$-th user can be written as $\mathbf{y}_{m}=\mathbf{h}_{m} * \mathbf{p}+\mathbf{w}_{m}$, where $\mathbf{w}_{m}=$ $\left[w_{m}(0), w_{m}(1), \cdots, w_{m}\left(L_{L C M}-1\right)\right]^{T}$ is the noise vector with $w_{m}(l) \sim \mathcal{C N}\left(0, \sigma^{2}\right)$.

At the receiver, $\mathbf{y}_{m}$ is split into $N_{s y m, k}$ non-overlapping SFMC symbols each being a length $L_{k}$ vector for receiver baseband processing. Let us define $\mathbf{p}_{i}$ and $\mathbf{w}_{m, i}$ as the $[(i-$ 1) $L_{k}+1$ ]-th to $i L_{k}$-th element of $\mathbf{p}$ and $\mathbf{w}_{m}$, respectively. Then the signal (before channel equalization) in the $i$-th symbol of the $m$-th user allocated to the $v$-th subband of the $k$-th service can be written as

$$
\mathbf{y}_{m, i}=\mathbf{D}_{k, v}^{H} \mathbf{R}_{k} \overline{\mathbf{A}}_{k, v}^{H} \mathbf{B}_{m}\left(\mathbf{p}_{i}+\mathbf{w}_{m, i}\right),
$$

where $\mathbf{R}_{k}=\left[\mathbf{0}_{N_{k} \times L_{C P, k}}, \mathbf{I}_{N_{k}}\right] \in \mathbb{R}^{N_{k} \times L_{k}}$ is matrix representation of $\mathrm{CP}$ removal operation. $\mathbf{B}_{m} \in \mathbb{C}^{L_{k} \times L_{k}}$ is the equivalent Toeplitz channel convolution matrix of $\mathbf{h}_{m}$, with first column $\left[\mathbf{h}_{m}, \mathbf{0}_{\left(L_{k}-L_{C H, m}-1\right) \times 1}\right]^{T}$ and first row $\left[h_{m}(0), \mathbf{0}_{\left(L_{k}-1\right) \times 1}\right]$.

Equation (5) is the frequency domain signal model before channel equalization. The normal frequency domain one-tap channel equalization algorithms such as zero-forcing ( $\mathrm{ZF}$ ) or minimum mean square error (MMSE) can be adopted to estimate the transmitted information symbols [9], and the details are omitted in the paper.

\section{MR MS-SFMC system model}

MR MS-SFMC varies the sampling rate in the baseband signal processing to benefit from low-complexity low-dimension DFT operation. The MR MS-SFMC system block diagram is shown in Fig. 4, where the normal CP-OFDM modulation is performed as its first step. Different from the SR MS-SFMC system that using a high-dimension IDFT spread matrix, MR MS-SFMC system uses $Z_{k}$-point DFT matrix. The signal in $i$ th symbol and $v$-th subband of the $k$-th service after the IDFT and $\mathrm{CP}$ insertion can be written in matrix form as follows

$$
\tilde{\mathbf{b}}_{k, v, i}=\tilde{\mathbf{C}}_{k} \tilde{\mathbf{D}}_{k, v} \mathbf{a}_{k, v, i}
$$

where $\tilde{\mathbf{D}}_{k, v}$ is full size $Z_{k}$-point DFT matrix and $\tilde{\mathbf{C}}_{k}=$ $\left[\mathbf{0}_{\tilde{L}_{C P, k} \times\left(Z_{k}-\tilde{L}_{C P, k}\right)}, \mathbf{I}_{\tilde{L}_{C P, k}} ; \mathbf{I}_{Z_{k}}\right] \in \mathbb{R}^{\left(Z_{k}+\tilde{L}_{C P, k}\right) \times Z_{k}}$ is the matrix form of the CP insertion operation with $\tilde{L}_{C P, k}=$ $\left\lceil L_{C P, k} / Q_{k}\right\rceil$ being the CP length of the $k$-th service $b e$ fore upsampling, $\left\lceil L_{C P, k} / Q_{k}\right\rceil$ denotes ceiling operations on $L_{C P, k} / Q_{k}$. The upsampling rate $Q_{k}$ can be written as

$$
Q_{k}=1 / B_{k} .
$$

Note that the subband signal $\tilde{\mathbf{b}}_{k, v, i}$ occupies the whole baseband bandwidth, the interpolation (upsampling) by a factor of $Q_{k}$ is required to map the signal to the corresponding bandwidth [16], following by an anti-aliasing filter. Then we have the filtered signal as

$$
\tilde{\mathbf{c}}_{k, v, i}=\mathbf{f}_{k, v} * \mathbf{Q}_{k} \tilde{\mathbf{b}}_{k, v, i}=\mathbf{A}_{k, v} \mathbf{Q}_{k} \tilde{\mathbf{b}}_{k, v, i},
$$

where $\mathbf{Q}_{k}$ is the interpolating matrix by upsampling $\tilde{\mathbf{b}}_{k, v, i}$ by a factor of $Q_{k}$. Note that after the upsampling, the sampling rate of the MR system is the same as the SR system.

Following the similar derivation as in SR system, by overlapping symbols to reconstruct the frame structure, the signal in $i$-th symbol and $v$-th subband of the $k$-th service can be written as

$$
\begin{array}{r}
\tilde{\mathbf{q}}_{k, v, i}=\frac{1}{\tilde{\rho}_{k, v}}\left(\overline{\mathbf{A}}_{k, v} \mathbf{Q}_{k} \tilde{\mathbf{b}}_{k, v, i}+\tilde{\mathbf{A}}_{k, v} \mathbf{Q}_{k} \tilde{\mathbf{b}}_{k, v, i-1}\right. \\
\left.+\hat{\mathbf{A}}_{k, v} \mathbf{Q}_{k} \tilde{\mathbf{b}}_{k, v, i+1}\right),
\end{array}
$$

where $\tilde{\rho}_{k, v}=\sqrt{\tilde{\mathbf{e}}_{k, v, i}^{H} \tilde{\mathbf{e}}_{k, v, i} / Z_{k}}$ is the transmission power normalization factor with $\tilde{\mathbf{e}}_{k, v, i}=\overline{\mathbf{A}}_{k, v} \mathbf{Q}_{k} \tilde{\mathbf{b}}_{k, v, i}+$ $\tilde{\mathbf{A}}_{k, v} \mathbf{Q}_{k} \tilde{\mathbf{b}}_{k, v, i-1}+\hat{\mathbf{A}}_{k, v} \mathbf{Q}_{k} \tilde{\mathbf{b}}_{k, v, i+1}$.

At the receiver side, as shown in Fig. 4, the received signal of the $k$-th service will be first downsampled by a factor of $Q_{k}$, followed by subband filtering, CP removal and DFT operation. Then the signal (before channel equalization) in the $i$-th symbol of the $m$-th user allocated to the $v$-th subband of the $k$-th service can be written as

$$
\tilde{\mathbf{y}}_{m, i}=\tilde{\mathbf{D}}_{k, v}^{H} \tilde{\mathbf{R}}_{k} \mathbf{Q}_{k}^{H} \overline{\mathbf{A}}_{k, v}^{H} \mathbf{B}_{m}\left(\tilde{\mathbf{p}}_{i}+\mathbf{w}_{m, i}\right),
$$

where $\tilde{\mathbf{p}}_{i}$ is the $\left[(i-1) L_{k}+1\right]$-th to $i L_{k}$-th element of $\tilde{\mathbf{p}}=\sum_{k=1}^{K} \sum_{v=1}^{V_{k}} \tilde{\mathbf{q}}_{k, v} \in \mathbb{C}^{L_{L C M} \times 1}$ and $\tilde{\mathbf{q}}_{k, v}=$ $\left[\tilde{\mathbf{q}}_{k, v, 1} ; \tilde{\mathbf{q}}_{k, v, 2} ; \cdots ; \tilde{\mathbf{q}}_{k, v, N_{s y m, k}}\right]$. Note that here $\mathbf{Q}_{k}^{H}$ means to downsample signal by a factor of $Q_{k}$ and $\tilde{\mathbf{R}}_{k}=$ $\left[\mathbf{0}_{Z_{k} \times \tilde{L}_{C P, k}}, \mathbf{I}_{Z_{k}}\right] \in \mathbb{R}^{Z_{k} \times\left(Z_{k}+\tilde{L}_{C P, k}\right)}$ is matrix representation of the CP' removal operation.

Similarly, one-tap channel equalization algorithms can be applied based on equation (17) to estimate the transmitted information symbols, and the details are omitted in the paper.

\section{COMPARISONS OF SR AND MR MS-SFMC SYSTEMS}

Comparing the SR and MR implementations illustrated in Fig. 3 and Fig. 4, the most significant difference between the two lies in the subband allocation and processing. SR MS-SFMC uses corresponding columns of the full-size IDFT matrix to assign the signals to its subband and the subband filter is adopted to isolate the signals between adjacent subbands in order to reduce the ISBI. On the other hand, the 


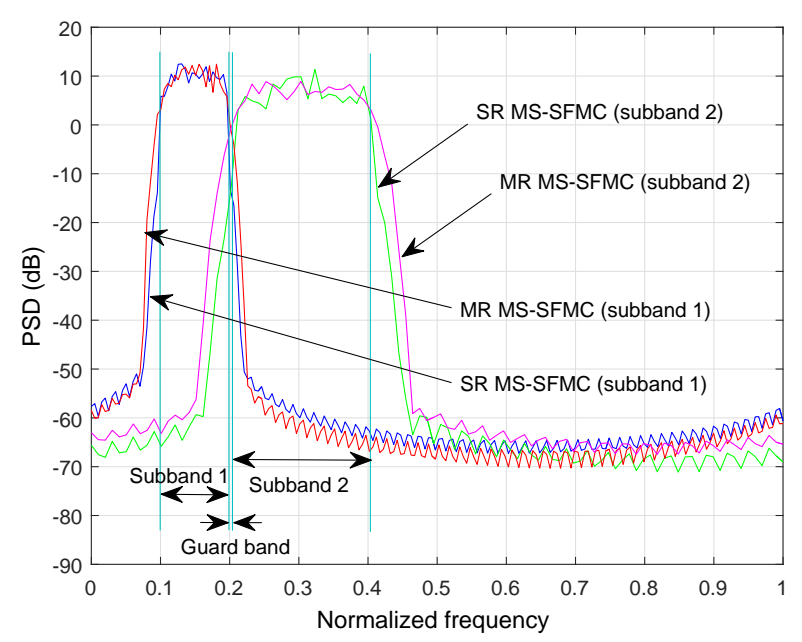

Fig. 5. PSD of SR and MR MS-SFMC systems.

MR MS-SFMC system in Fig. 4 uses low-dimension and lowcomplexity full size IDFT matrix that spreads the signal into the whole baseband bandwidth. The following upsampling operation maps the signal into the allocated subband. It creates image signals in adjacent bands as a side-effect, leading to increased the $\mathrm{OoB}$ emission and ISBI levels and degraded performance in comparison with the SR implementation for a given filter.

In what follows, we will compare the SR and MR implementations in terms of $\mathrm{OoB}$ emission and computational complexity.

\section{A. OoB emission and ISBI}

To illustrate the OoB emission level and the ISBI of the two implementations, Fig. 5 compared the power spectrum density (PSD) of the SR and MR MS-SFMC systems by considering two subbands with $B_{1}=0.1$ and $B_{2}=0.2$ and both subbands contain 20 subcarriers. In addition, the guard band $\Delta B=\left[\Delta B_{0}, \Delta B_{1}, \Delta B_{2}\right]=[0.1,0.01,0.61]$. Note that the guard band between the two adjacent subband $\Delta B_{1}=0.01$ is equal to 2 or 1 subcarriers of the first and second subband, respectively. We use the soft truncated Sinc prototype filter with the filter length equals to half symbol duration [11], [4].

From Fig. 5, the MR system shows slightly worse performance in terms of $\mathrm{OoB}$ emission level due to the residual image signals, resulting in a larger ISBI that may cause performance loss in terms of bit error rate (BER) and mean square error (MSE) for a given guard band bandwidth, which will be illustrated in the simulation section.

\section{B. Complexity}

Comparing with the SR system, the MR system benefits from lower dimension DFT / IDFT operations, resulting in significant computational complexity reduction. In the real implementations, the DFT / IDFT size can take a power of 2 to facilitate the FFT / IFFT algorithms. A $N$-point FFT / IFFT has the complexity of $C(N)=N \log _{2} N-$
$3 N+4$ [17]. For a SR MS-SFMC system comprising of $V=\sum_{k=1}^{K} V_{k}$ subbands, $V$ times IFFT are performed and the IFFT size for the $k$-th service is $M_{k}$. The total complexity for IFFT operation can be calculated as $\sum_{k=1}^{K} V_{k} C\left(N_{k}\right)=$ $\sum_{k=1}^{K} V_{k}\left(N_{k} \log _{2} N_{k}-3 N_{k}+4\right)$. The subband filtering can be performed by linear convolution with the total complexity of $\sum_{k=1}^{K} V_{k} L_{k} L_{F, k}$. In total, the transmitter complexity is $\sum_{k=1}^{K} V_{k}\left(N_{k} \log _{2} N_{k}-3 N_{k}+4+L_{k} L_{F, k}\right)$. The receiver has the same complexity, resulting in the total complexity of SR MS-SFMC as $\sum_{k=1}^{K} 2 V_{k}\left(N_{k} \log _{2} N_{k}-3 N_{k}+4+L_{k} L_{F, k}\right)$.

On the other hand, the FFT/IFFT complexity of the total $V$ subbands in the MR MS-SFMC system is $\sum_{k=1}^{K} V_{k} C\left(Z_{k}\right)=$ $\sum_{k=1}^{K} V_{k}\left(Z_{k} \log _{2} Z_{k}-3 Z_{k}+4\right)$. Note that the filtering operation complexity in the MR MS-SFMC system is $\sum_{k=1}^{K} V_{k}\left(Z_{k}+\tilde{L}_{C P, k}\right) L_{F, k}$. The total complexity of the MR MS-SFMC transceiver is $\sum_{k=1}^{K} 2 V_{k}\left[Z_{k} \log _{2} Z_{k}-3 Z_{k}+4+\right.$ $\left.\left(Z_{k}+\tilde{L}_{C P, k}\right) L_{F, k}\right]$.

According to equation (5), we have $N_{k}=M_{k} / B_{k}=$ $Z_{k} V_{k} / B_{k}$. When the number of subbands in one service is larger or the each service only occupies small percentage of the whole bandwidth, we have $V_{k} / B_{k}>>1$, i.e., $Z_{k}<<N_{k}$. In this case, the MR system has much less complexity than the SR system.

\section{Simulations}

In this section, we compare the performance of the proposed SR MS-SFMC and MR MS-SFMC systems. The signal is modulated using 16-QAM (Quadrature amplitude modulation) with power normalized to unity and the input SNR is controlled by the noise variance. We adopt soft truncated Sinc filter [11], [4] with the filter length being the half of the symbol duration for each service. We consider two subbands with each consisting of 20 subcarriers and $B_{1}=0.1$ and $B_{2}=0.2$. i.e., $\Delta f_{1}=0.005$ and $\Delta f_{2}=0.01$. A one-tap MMSE channel equalization algorithm is adopted to all cases.

We first investigate the impact of ISBI on different subcarriers in terms of MSE performance with $\Delta B_{1}=0$ and $\Delta B_{1}=0.01$ respectively, for both SR and MR MS-SFMC systems. A noise-free and single-path channel is considered in this simulation to focus on the interference analysis only, while a general case will be considered in the next simulation.

Fig. 6 shows that MSE varies along the subcarrier index for all considered cases. Specifically, the subcarriers at the edge of subbands have higher MSE than in the middle due to two reasons: 1) filter response is frequency selective with larger gain in the middle and smaller gain in the edges of a subband [9]; 2) the subcarriers close to the edge of subband suffers from larger ISBI. Comparing the SR with MR systems, the SR based system shows better performance in terms of MSE for a given guard band. While a larger guard band can effectively reduce the MSE for both implementations.

Next, we investigate the impact of different guard band between two subbands on the BER performance of the two subbands in LTE extended typical urban (ETU) channels. As shown in Fig.7, the BER performance reduces significantly when the guard band bandwidth increases from $\Delta B_{1}=0$ 


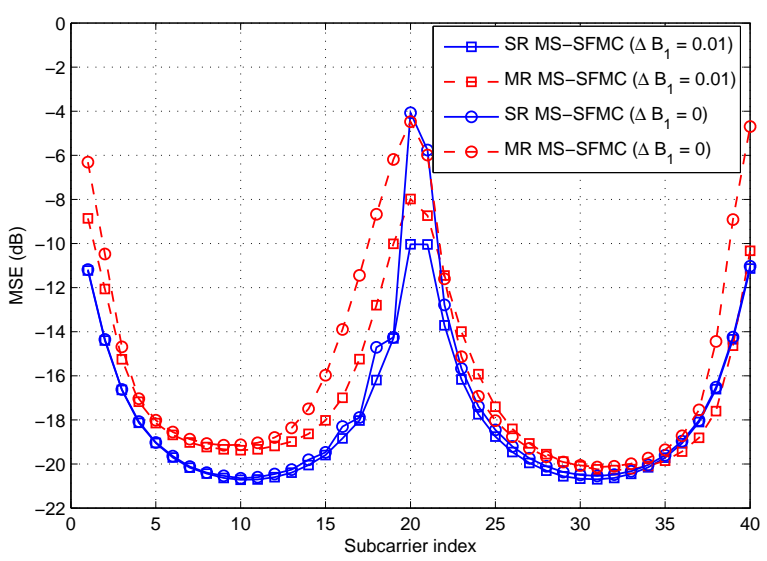

Fig. 6. MSE versus subcarrier index for different guard band in SR and MR MS-SFMC systems.

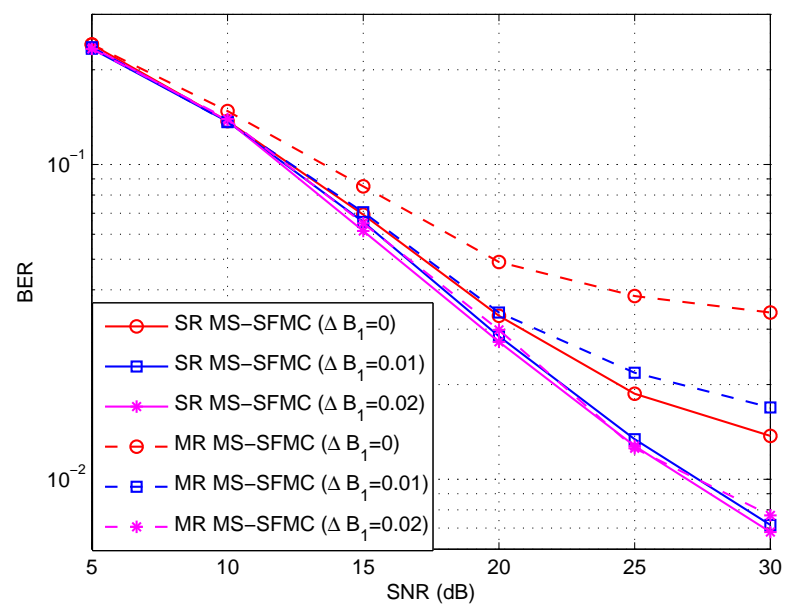

Fig. 7. BER versus input SNR for different guard band in SR and MR MSSFMC systems.

to $\Delta B_{1}=0.01$ for both implementations. However, the gain is not significant when the guard band is further increased from $\Delta B_{1}=0.01$ to $\Delta B_{1}=0.02$, which implies that a couple of subcarriers as guard band are sufficient to isolate ISBI between the two subbands. Comparing the SR and MR implementations, as discussed in the Section III, the SR based system outperforms MR based system in all case in terms of BER at the cost of higher computational complexity.

\section{CONCLUSIONS AND FUTURE WORKS}

A framework for $5 \mathrm{G}$ multi-service subband filtered multicarrier systems has been established in this paper. Two subband filtering implementations: SR and MR MS-SFMC systems were proposed. The system models and the pros and cons of them were discussed in terms of the performance and computational complexity. Specifically, the SR system outperforms the MR system in terms of performance while the MR system has a significantly reduced computational complexity. Numerical results were presented to demonstrate the effectiveness of the analysis and proposed systems.

This paper provides a general framework for MS-SFMC based system design, algorithm design and performance analysis. Future work can be focused on the following topics: a) theoretical performance analysis for both SR and MR systems to derive the performance and capacity bounds; b) design of ISBI cancelation algorithms with reasonable computational complexity for both implementations; c) filter optimization to maximize the capacity by minimizing the inter-carrier interference and inter-symbol interference.

\section{ACKNOWLEDGMENT}

The authors would like to acknowledge the support of the University of Surrey 5GIC (http://www.surrey.ac.uk/5gic) members for this work.

\section{REFERENCES}

[1] Cisco, "Cisco visual networking index: Global mobile data traffic forecast update, 2015-2020," Tech. Rep., 2016.

[2] A. Ijaz, L. Zhang, M. Grau, A. Mohamed, S. Vural, A. U. Quddus, M. A. Imran, C. Foh, and R. Tafazolli, "Enabling massive IoT in 5G and beyond systems: PHY radio frame design considerations," IEEE Access, 2016.

[3] 5GNOW, "D3.2: 5G waveform candidate selection," Tech. Rep., 2014.

[4] X. Zhang, M. Jia, L. Chen, J. Ma, and J. Qiu, "Filtered-OFDM - enabler for flexible waveform in the 5th generation cellular networks," in IEEE Global Communications Conference (GLOBECOM), Dec 2015, pp. 1-6.

[5] A. Ijaz, L. Zhang, A. Quddus, and R. Tafazolli, "HARQ in relay assisted transmission for machine type communications," IEEE Wireless Communications Letters, p. 172 175, April 2016.

[6] P. Xiao, C. Toal, D. Burns, V. Fusco, and C. Cowan, "Transmit and receive filter design for ofdm based wlan systems," in IEEE WCSP, 2010.

[7] G. Wunder, P. Jung, M. Kasparick, T. Wild, F. Schaich, Y. Chen, S. Brink, I. Gaspar, N. Michailow, A. Festag, L. Mendes, N. Cassiau, D. Ktenas, M. Dryjanski, S. Pietrzyk, B. Eged, P. Vago, and F. Wiedmann, "5GNOW: non-orthogonal, asynchronous waveforms for future mobile applications," IEEE Communications Magazine, vol. 52, no. 2, pp. 97-105, February 2014.

[8] V. Vakilian, T. Wild, F. Schaich, S. Ten Brink, and J.-F. Frigon, "Universal-filtered multi-carrier technique for wireless systems beyond LTE," in IEEE Globecom Workshops (GC Wkshps), 2013, pp. 223-228.

[9] L. Zhang, A. Ijaz, P. Xiao, M. Ali Imran, and R. Tafazolli, "MU-UFMC system performance analysis and optimal filter length and zero padding length design," submitted to IEEE Transactions on Communications, 2016.

[10] L. Zhang, P. Xiao, and A. Quddus, "Cyclic prefix based universal filtered multi-carrier system and performance analysis," IEEE Signal Processing Letters, 2016.

[11] J. Abdoli, M. Jia, and J. Ma, "Filtered ofdm: A new waveform for future wireless systems," in IEEE Signal Processing Advances in Wireless Communications (SPAWC), June 2015, pp. 66-70.

[12] B. Farhang-Boroujeny, "OFDM versus filter bank multicarrier," IEEE Signal Processing Magazine, vol. 28, no. 3, pp. 92-112, May 2011.

[13] R. Razavi, P. Xiao, and R. Tafazolli, "Information theoretic analysis of OFDM/OQAM with utilized intrinsic interference," in IEEE Signal Processing Letters, May 2015, pp. 618-622.

[14] J. Du, P. Xiao, J. Wu, and Q. Chen, "Design of isotropic orthogonal transform algorithm-based multicarrier systems with blind channel estimation," IET Communications, vol. 6, pp. 2695-2704, November 2012.

[15] G. Fettweis, M. Krondorf, and S. Bittner, "GFDM - generalized frequency division multiplexing," in IEEE Vehicular Technology Conference, 2009, April 2009, pp. 1-4.

[16] P. Vaidyanathan, "Multirate systems and filter banks," Prentice Hall, 1993.

[17] H. Malvar, Signal Processing with Lapped Transforms. Artech House, 1992. 